\title{
DECODING SATELLITE IMAGES OF EARTHQUAKES, PART-V: APPROXIMATING DERIVATIVES BY CONVOLUTIONS
}

\section{Saumik Dana ${ }^{1, *}$}

${ }^{1}$ University of Southern California, Los Angeles, CA 90089

*Address all correspondence to: Saumik Dana, E-mail: sdana@usc.edu

This document is part $V$ of a series of documents providing a lowdown on image processing in the context of understanding satellite images of earthquakes.

KEY WORDS: Earthquakes, satellite images, image processing, derivatives, convolutions

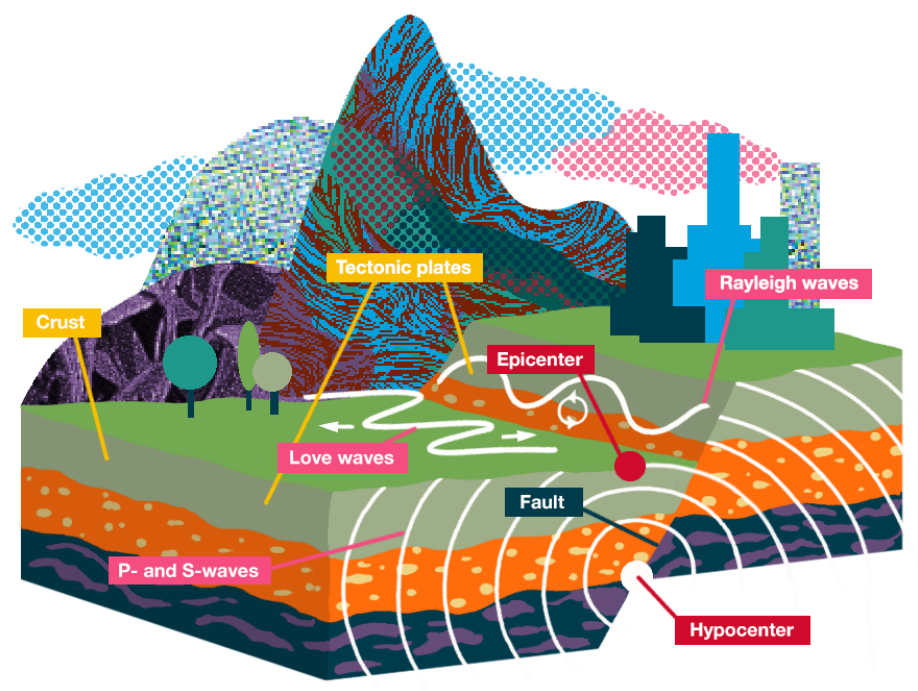

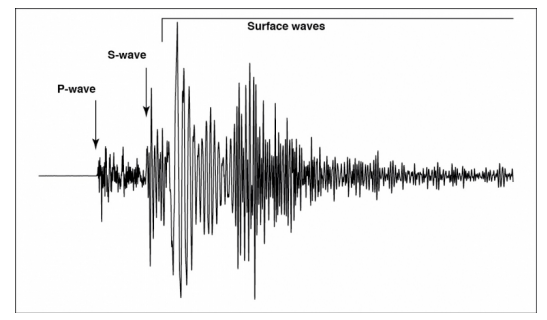

(b) P-, S- and surface waves

(a) Propagation of waves

FIG. 1: Source: Caltech Science Exchange

The earthquake cycle, from slow deformation associated with interseismic behavior to rapid deformation associated with earthquake rupture, spans spatial scales ranging from fractions of a meter associated with the size of contact asperities on faults and individual grains to hundreds of kilometers associated with plate boundaries [1]. Similarly, temporal scales range from fractions of a second associated with slip at a point during earthquake rupture to hundreds of years of strain accumulation between earthquakes. In many cases, earthquakes are triggered after pore pressure perturbations activate critically stressed seismogenic faults [1], not just due to natural causes like earth tides [2], rainfall [3], snowfall [4], typhoons [5], but also due to human activity [6]. Ground motion and failure due to earthquakes lead to huge losses to the tune of billions of dollars annually in the United States (see Fig. 2). Understanding the causality between the events leading to fault slip, the location of the slipping fault and the earthquake recording is 


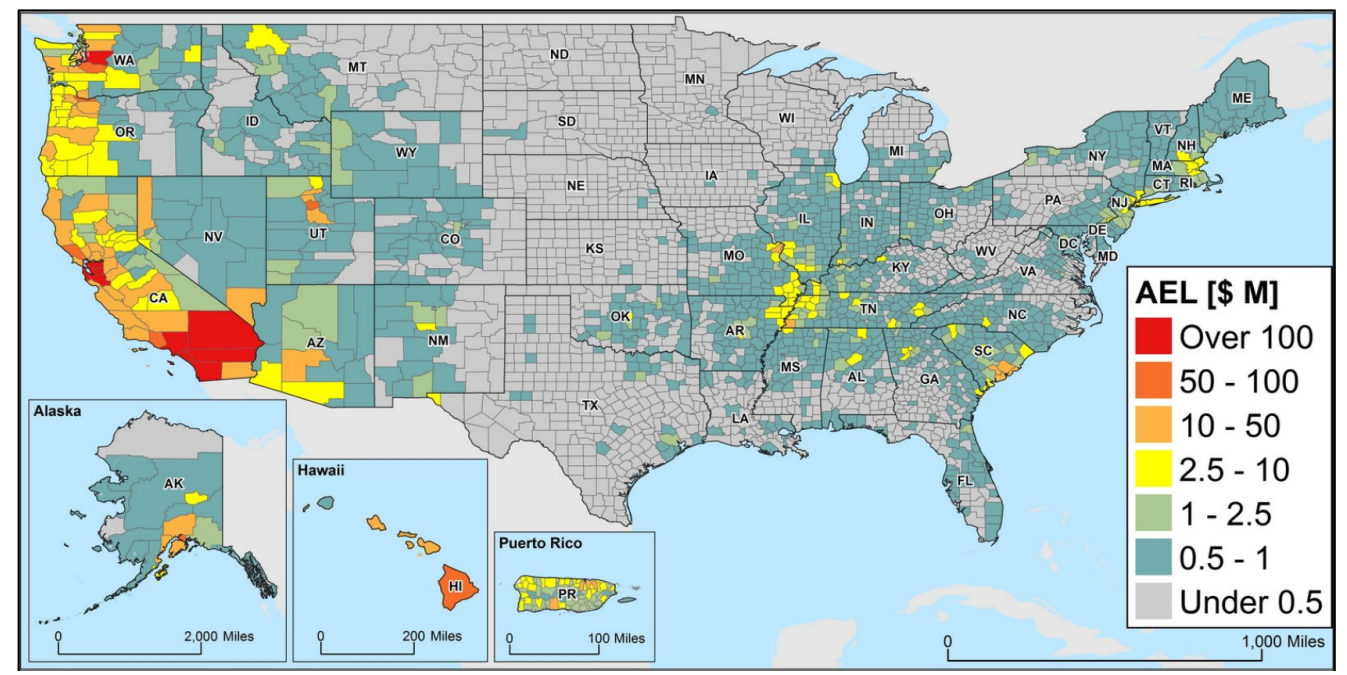

FIG. 2: Annualized Earthquake Losses (AEL: estimated long-term value of earthquake losses to the general building stock) by County. Sources: (1) https://www.usgs.gov/news/usgs-collaborates-fema-national-earthquake-loss-estimate and (2) https://www.fema.gov/hazus. The analysis yielded an estimate of the national AEL of $\$ 6.1$ billion per year. Loss estimates based on the best science and engineering available (during 2016-2017). The study only addresses direct economic losses to buildings, and does not cover damage and losses to critical facilities, transportation and utility lifelines or indirect economic losses.

important for seismic design and monitoring of underground structures [7], bridges [8] and reinforced concrete buildings [9] as well as climate mitigation projects like carbon sequestration [10] and energy technologies like enhanced geothermal systems [11] or oilfield wastewater disposal [12]. A holistic framework would be to link the forward modeling framework going from quasi-static [13-25] to fully dynamic to the inverse modeling framework in the realm of remote sensing and image processing.

\subsection{Deep learning to uncover partial differential equations}

The most critical aspect of image processing is the approximation of derivatives by convolution operations. In this document, we give a lowdown on how derivatives convert into convolutions, and how this simple operation is a game-changer in the realm of fusing computer vision with forward modeling. The reason is "convolution is the basis of convolutional neural networks, and these neural networks can be used to construct PDEs from data". This is incredibly important going forward as the "existing intellectual capital is not enough to uncover governing partial differential equations for new phenomena, and hence data will guide us along the way in constructing these governing differential equations". We make some key points here:

$\checkmark$ With new phenomena being discovered and researched, it is next to impossible to know the partial differential equation governing the phenomena apriori

$\checkmark$ We have relied on phenomenological studies and proofs of the scientists in the post-renaissance Europe to guide us to using certain oft-repeated partial differential equations for all phenomena

$\checkmark$ These PDEs, although incredibly robust for phenomena hitherto, cannot possibly encompass new phenomena as they are uncovered by mankind

$\checkmark$ If the PDEs are insufficient, no forward modeling procedure in useful, as the numerical methods are only as accurate as the PDE itself

$\checkmark$ With the ubiquity of neural networks in scientific community, and the advent of high performance computing, it is now possible to uncover governing partial differential equations purely from data, and the tool that is used to do this unraveling is deep learning

$\checkmark$ "The differential operators are basically treated as neurons in the neural network, and the action of the neuron i.e. differential operator is treated as a convolution operation" 
$\checkmark$ "In this realm, convolutional neural networks come to be deployed, and deep learning spits out a combination of differential operators along with coefficients, which is the PDE being uncovered"

To formally put it in mathematical terms, we refer to the work of [26], which expresses the PDE to be uncovered as a function of the many differential operators that are encompassed in the realm of possibility. Let us look at a two-dimensional phenomena, with the spatial variables being $x$ and $y$, and let

$$
u_{t}=\mathcal{F}
$$

be the PDE being uncovered for the primary variable $u$. Let us work on the assumption that the "phenomena is a second order phenomena (that is where the only amount of intuition that is needed comes in)", and let us express $\mathcal{F}$ as a function of all possible derivatives upto the second order as follows:

$$
u_{t}(t, x, y)=\mathcal{F}\left(x, y, u, u_{x}, u_{y}, u_{x} u_{y}, u_{x x}, u_{y y}, u_{x x} u_{y y}\right) \equiv A x+B y+C u+D u_{x}+\cdots
$$

"The idea is to fit the data to $\mathcal{F}$ to determine the coefficients $A, B, \cdots$ using deep learning on convolutional neural networks" by replacing the derivatives by convolution operations as follows

$$
u_{t}(t, x, y)=\mathcal{F}\left(x, y, D_{00} \circledast u, D_{10} \circledast u, D_{20} \circledast u, \cdots\right) \equiv A x+B y+C D_{00} \circledast u+D D_{10} \circledast u+\cdots
$$

where $D_{00}, D_{10}$, and so on, are convolution operators such that

$$
D_{i j} \circledast u \equiv \frac{\partial^{i+j} u}{\partial^{i} x \partial^{j} y}
$$

The most important thing to understand now is the connection between derivatives and convolutions, for which we take a deep dive into the intellectual capital of computer vision [27]

\section{GOING FROM DERIVATIVES TO DIFFERENCING TO CONVOLUTION}

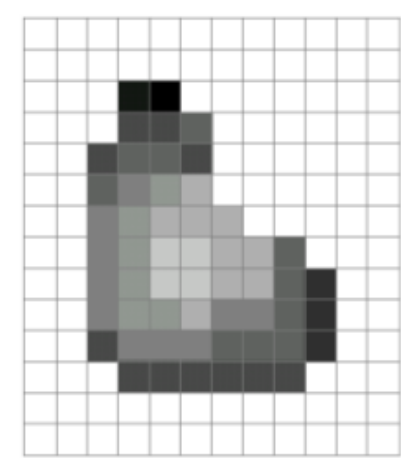

\begin{tabular}{|l|l|l|r|r|r|r|r|r|r|r|r|}
\hline 255 & 255 & 255 & 255 & 255 & 255 & 255 & 255 & 255 & 255 & 255 & 255 \\
\hline 255 & 255 & 255 & 255 & 255 & 255 & 255 & 255 & 255 & 255 & 255 & 255 \\
\hline 255 & 255 & 255 & 20 & 0 & 255 & 255 & 255 & 255 & 255 & 255 & 255 \\
\hline 255 & 255 & 255 & 75 & 75 & 75 & 255 & 255 & 255 & 255 & 255 & 255 \\
\hline 255 & 255 & 75 & 95 & 95 & 75 & 255 & 255 & 255 & 255 & 255 & 255 \\
\hline 255 & 255 & 96 & 127 & 145 & 175 & 255 & 255 & 255 & 255 & 255 & 255 \\
\hline 255 & 255 & 127 & 145 & 175 & 175 & 175 & 255 & 255 & 255 & 255 & 255 \\
\hline 255 & 255 & 127 & 145 & 200 & 200 & 175 & 175 & 95 & 255 & 255 & 255 \\
\hline 255 & 255 & 127 & 145 & 200 & 200 & 175 & 175 & 95 & 47 & 255 & 255 \\
\hline 255 & 255 & 127 & 145 & 145 & 175 & 127 & 127 & 95 & 47 & 255 & 255 \\
\hline 255 & 255 & 74 & 127 & 127 & 127 & 95 & 95 & 95 & 47 & 255 & 255 \\
\hline 255 & 255 & 255 & 74 & 74 & 74 & 74 & 74 & 74 & 255 & 255 & 255 \\
\hline 255 & 255 & 255 & 255 & 255 & 255 & 255 & 255 & 255 & 255 & 255 & 255 \\
\hline 255 & 255 & 255 & 255 & 255 & 255 & 255 & 255 & 255 & 255 & 255 & 255 \\
\hline
\end{tabular}

FIG. 3: An image is a grid of pixels. 0 is for black and 255 is for white on the grayscale scale.

As shown in Fig. 3, an image is a grid (matrix) of intensity values. To convert the image to a signal, we can think of a (grayscale) image as a function, $f$, from $R^{2}$ to $R$ (or a 2D signal): $f(x, y)$ gives the intensity at position $(x, y)$. A digital image is a discrete (sampled, quantized) version of this function. 


\subsection{Linear filtering}

\begin{tabular}{|l|l|l|l|l|l|l|l|l|l|}
\hline 45 & 60 & 98 & 127 & 132 & 133 & 137 & 133 \\
\hline 46 & 65 & 98 & 123 & 126 & 128 & 131 & 133 \\
\hline 47 & 65 & 96 & 115 & 119 & 123 & 135 & 137 \\
\hline 47 & 63 & 91 & 107 & 113 & 122 & 138 & 134 \\
\hline 50 & 59 & 80 & 97 & 110 & 123 & 133 & 134 \\
\hline 49 & 53 & 68 & 83 & 97 & 113 & 128 & 133 \\
\hline 50 & 50 & 58 & 70 & 84 & 102 & 116 & 126 \\
\hline 50 & 50 & 52 & 58 & 69 & 86 & 101 & 120 \\
\hline
\end{tabular}

FIG. 4: Neighborhood filtering (convolution): The image on the left is convolved with the filter in the middle to yield the image on the right. The light blue pixels indicate the source neighborhood for the light green destination pixel. Source: [27]

The most commonly used type of neighborhood operator is a linear filter, in which an output pixel's value is determined as a weighted sum of input pixel values (Fig. 4),

$$
g(i, j)=\sum_{k, l} h(k, l) f(i-k, j-l)
$$

The entries in the weight kernel or mask $h(k, l)$ are often called the filter coefficients. This is called the convolution operator,

$$
g=h \circledast f
$$

This operation has a lot of similarity with the idea of "stencils in finite differencing".

\subsection{Partial derivative}

The partial derivative of a continuous function $f(x, y)$ with respect to the variable $x$ is defined as the local slope of the plot of the function along the $x$ direction or, formally, by the following limit:

$$
\frac{\partial f}{\partial x}=\lim _{\Delta x \rightarrow 0} \frac{f(x+\Delta x, y)-f(x, y)}{\Delta x}
$$

If the granularity of the data in the $x$ direction is $\Delta x$, then:

$$
\frac{\partial f}{\partial x} \approx \frac{f(x+\Delta x, y)-f(x, y)}{\Delta x}
$$

As shown in Fig. 5, the first derivative can be approximated by a convolution with mask $h(x, y)$, with the formal mathematical expression given by

$$
\frac{\partial f}{\partial x} \approx \frac{f(x+\Delta x, y)-f(x, y)}{\Delta x} \equiv \frac{1}{\Delta x} h \circledast f
$$

Other derivatives like $\frac{\partial f}{\partial y}$, and so on, can be expressed as convolution operations 

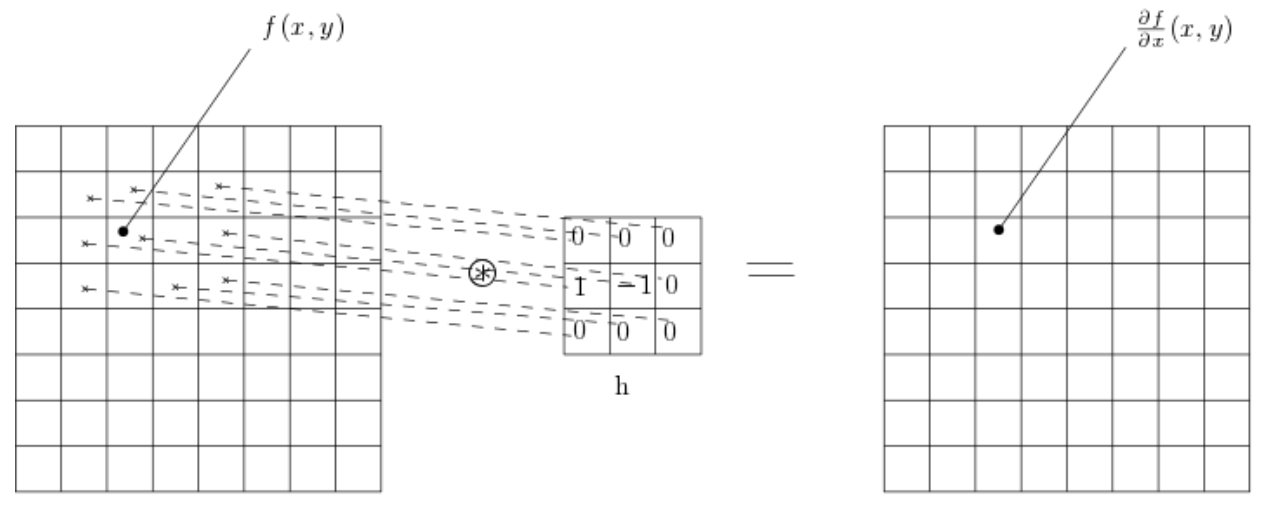

f

$\frac{\partial f}{\partial x}$

FIG. 5: The first derivative is equivalent convolution with mask $h$

\subsection{Moving onto continuous functions}

Eq. (2) for discrete functions can be written for continuous functions as:

$$
g(x, y)=\int_{x^{\prime}} \int_{y^{\prime}} h\left(x^{\prime}, y^{\prime}\right) f\left(x-x^{\prime}, y-y^{\prime}\right) \equiv h \circledast f
$$

If the granularity of the data in the $x$ direction is $d x$, then:

$$
\frac{\partial f}{\partial x} \approx \frac{f(x+d x, y)-f(x, y)}{d x}
$$

From Eqs. (2) and (3), it is clear that a function $h(x, y)$ which satisfies:

$$
h(x, y)= \begin{cases}1 & \text { if } \quad x=-d x \\ -1 & \text { if } \quad x=0 \\ 0 & \text { otherwise }\end{cases}
$$

would be a mask for the first partial derivative approximated as a convolution. The mask in Eq. (5) has the undesirable property of the derivative blowing up at $x=-d x$ and $x=0$, and it is common to approximate the higher order derivatives of $f$ as derivatives of the mask $h$ convolving with $f$. In lieu of that, "the discrete mask type function is replaced by a Gaussian function (also called as Gaussian filter in digital signal processing)" as follows:

$$
h(x, y)= \begin{cases}\gamma(x, y) & \text { if } \quad x=-d x \\ \gamma(x, y) & \text { if } \quad x=0 \\ 0 & \text { otherwise }\end{cases}
$$

where the generic form of the Gaussian function is:

$$
\gamma:=\frac{1}{2 \pi \sigma^{2}} e^{-\frac{x^{2}+y^{2}}{2 \sigma^{2}}}
$$

Needless to say, the Gaussian function is the most popular filter in computer vision [27] and signal processing [28] due to multiple desirable properties. 


\section{References}

[1] Hiroo Kanamori and Emily E Brodsky. The physics of earthquakes. Reports on Progress in Physics, 67(8):1429, 2004.

[2] Christopher H Scholz, Yen Joe Tan, and Fabien Albino. The mechanism of tidal triggering of earthquakes at mid-ocean ridges. Nature communications, 10(1):1-7, 2019.

[3] Sebastian Hainzl, Toni Kraft, Joachim Wassermann, Heiner Igel, and E Schmedes. Evidence for rainfall-triggered earthquake activity. Geophysical Research Letters, 33(19), 2006.

[4] EK Montgomery-Brown, David R Shelly, and Paul A Hsieh. Snowmelt-triggered earthquake swarms at the margin of long valley caldera, california. Geophysical Research Letters, 46(7):3698-3705, 2019.

[5] ChiChing Liu, Alan T Linde, and I Selwyn Sacks. Slow earthquakes triggered by typhoons. Nature, 459(7248):833-836, 2009.

[6] Gillian R Foulger, Miles P Wilson, Jon G Gluyas, Bruce R Julian, and Richard J Davies. Global review of human-induced earthquakes. Earth-Science Reviews, 178:438-514, 2018.

[7] Youssef MA Hashash, Jeffrey J Hook, Birger Schmidt, I John, and Chiang Yao. Seismic design and analysis of underground structures. Tunnelling and underground space technology, 16(4):247-293, 2001.

[8] MJ Nigel Priestley, Frieder Seible, and Gian Michele Calvi. Seismic design and retrofit of bridges. John Wiley \& Sons, 1996.

[9] Jack Moehle. Seismic design of reinforced concrete buildings. McGraw-Hill Education, 2015.

[10] Mark D Zoback and Steven M Gorelick. Earthquake triggering and large-scale geologic storage of carbon dioxide. Proceedings of the National Academy of Sciences, 109(26):10164-10168, 2012.

[11] William L Ellsworth, Domenico Giardini, John Townend, Shemin Ge, and Toshihiko Shimamoto. Triggering of the pohang, korea, earthquake ( $\mathrm{m}$ w 5.5) by enhanced geothermal system stimulation. Seismological Research Letters, 90(5):1844-1858, 2019.

[12] Katie M Keranen and Matthew Weingarten. Induced seismicity. Annual Review of Earth and Planetary Sciences, 46:149$174,2018$.

[13] Saumik Dana and Mary F Wheeler. Augmented lagrangian for treatment of hanging nodes in hexahedral meshes. arXiv preprint arXiv:1809.04031, 2018.

[14] Saumik Dana and Karthik Reddy Lyathakula. Uncertainty quantification in friction model for earthquakes using bayesian inference. arXiv preprint arXiv:2104.11156, 2021.

[15] S. Dana and M. F. Wheeler. Convergence analysis of fixed stress split iterative scheme for anisotropic poroelasticity with tensor biot parameter. Computational Geosciences, 22(5):1219-1230, 2018.

[16] S. Dana and M. F. Wheeler. Convergence analysis of two-grid fixed stress split iterative scheme for coupled flow and deformation in heterogeneous poroelastic media. Computer Methods in Applied Mechanics and Engineering, 341:788-806, 2018 .

[17] Saumik Dana and Mary F Wheeler. Design of convergence criterion for fixed stress split iterative scheme for small strain anisotropic poroelastoplasticity coupled with single phase flow. arXiv preprint arXiv:1912.06476, 2019.

[18] Saumik Dana and Mary F Wheeler. An efficient algorithm for numerical homogenization of fluid filled porous solids: part-i. arXiv preprint arXiv:2002.03770, 2020.

[19] Saumik Dana and Mary F Wheeler. A machine learning accelerated $\mathrm{fe}^{2}$ homogenization algorithm for elastic solids. arXiv preprint arXiv:2003.11372, 2020.

[20] Saumik Dana, Benjamin Ganis, and Mary F. Wheeler. A multiscale fixed stress split iterative scheme for coupled flow and poromechanics in deep subsurface reservoirs. Journal of Computational Physics, 352:1-22, 2018. 
[21] Saumik Dana, Shriram Srinivasan, Satish Karra, Nataliia Makedonska, Jeffrey D Hyman, Daniel O’Malley, Hari Viswanathan, and Gowri Srinivasan. Towards real-time forecasting of natural gas production by harnessing graph theory for stochastic discrete fracture networks. Journal of Petroleum Science and Engineering, 195:107791, 2020.

[22] Saumik Dana, Joel Ita, and Mary F Wheeler. The correspondence between voigt and reuss bounds and the decoupling constraint in a two-grid staggered algorithm for consolidation in heterogeneous porous media. Multiscale Modeling \& Simulation, 18(1):221-239, 2020.

[23] Saumik Dana. A simple framework for arriving at bounds on effective moduli in heterogeneous anisotropic poroelastic solids. arXiv preprint arXiv:1912.10835, 2019.

[24] Saumik Dana. System of equations and staggered solution algorithm for immiscible two-phase flow coupled with linear poromechanics. arXiv preprint arXiv:1912.04703, 2019.

[25] S. Dana. Addressing challenges in modeling of coupled flow and poromechanics in deep subsurface reservoirs. PhD thesis, The University of Texas at Austin, 2018.

[26] Zichao Long, Yiping Lu, Xianzhong Ma, and Bin Dong. Pde-net: Learning pdes from data. In International Conference on Machine Learning, pages 3208-3216. PMLR, 2018.

[27] Richard Szeliski. Computer vision: algorithms and applications. Springer Science \& Business Media, 2010.

[28] Steven W Smith et al. The scientist and engineer's guide to digital signal processing. 1997. 